Proposal of mechanics of $\mathrm{I}$ i qui d crystal $\mathrm{s}$ and devel opment of I i quid crystal I i ne mi cr oact uat or $\mathrm{s}$

\begin{tabular}{|l|l|}
\hline 著者 & Chono Shi geom, Tsuj i Tomohi ro \\
\hline $\begin{array}{l}\text { j our nal or } \\
\text { publ i cat i on t i t l e }\end{array}$ & Appl i ed Physi cs Let ter s \\
\hline vol une & 92 \\
\hline number & 5 \\
\hline page r ange & $051905-1-051905-3$ \\
\hline year & 2008- 02 \\
\hline URL & ht t p: //hdl . handl e. net /10173/586 \\
\hline
\end{tabular}




\title{
Proposal of mechanics of liquid crystals and development of liquid crystalline microactuators
}

\author{
Shigeomi Chono a) and Tomohiro Tsuji \\ Department of Mechanical Engineering, Kochi University of Technology, Kami-shi, Kochi 782-8502, Japan
}

(Received 22 October 2007; accepted 9 January 2008; published online 5 February 2008)

\begin{abstract}
We propose a research field "mechanics of liquid crystals," in which liquid crystals are studied from a mechanical viewpoint. The unsteady behaviors of a liquid crystal between parallel plates under an electric field are investigated. The imposition of the electric field on the liquid crystal induces flows, whose profile and magnitude strongly depend on the twist angle of the director at the plates. A visualization experiment confirms the generation of flows. The mechanism of such generation can be explained by considering that the rotation of molecules generated by the imposition of the electric field induces a local velocity gradient. (C) 2008 American Institute of Physics.
\end{abstract}

[DOI: $10.1063 / 1.2840673$ ]

Since the discovery of the applicability of liquid crystals to display devices in the 1960s, many studies on liquid crystals have been carried out both fundamentally and practically. As a result, liquid crystal displays have established a strong position as a substitute for cathode ray tube displays. The manufacturing of such displays has grown into a large industry with a global scale and further development is expected. However, the technical development has reached a plateau except for a few trivial improvements.

A research field, such as "mechanics of liquid crystals," should exist and various studies concerning liquid crystals should be performed from a mechanical viewpoint, because in mechanical engineering, solid mechanics and fluid mechanics are established in the fields of solid, and gas and liquid, respectively. However, there is no such research field, while there are some studies where a liquid crystal is regarded as a fluid. ${ }^{1-4}$ Figure 1 shows the conceptual applications of liquid crystals; that is, we input some factors, such as electromagnetic field, flow, heat, and light, into liquid crystals and output different factors by utilizing the easy change in orientation state, which is the most representative feature of liquid crystals. An example of such applications is a liquid crystal display in which an electric field is input and a change in transmitted light is output. We can regard a liquid crystal as a medium of converting energy by combining input and output factors appropriately. The applications of liquid crystals are assumed to increase by changing the combination of input and output factors or by proposing other factors.

Although it is well known that the imposition of a velocity gradient makes liquid crystalline molecules rotate and reorient to a different direction, its reverse phenomenon, such that the rotation of the molecules (by imposing, for example, an electric field) induces a velocity gradient, is not clearly understood. Since this phenomenon corresponds to the energy conversion from electrical to kinetic energies, to utilize such induced flow leads to development of microactuators. Some studies ${ }^{5,6}$ were made for the generation of a flow, which was called "back flow;" however, in these studies, the mechanical applications of the back flow were not considered, although its effect on molecular orientation was investigated. In this letter, as a fundamental study for devel- oping microactuators driven by liquid crystals, we report computational results for the induced flow using the LeslieEricksen theory, ${ }^{7-9}$ which has been widely used as a constitutive equation for low-molar-mass nematic liquid crystals. We also refer to a visualization experiment and the mechanism of the flow generation.

The isothermal flow of liquid crystals under an electric field is governed by continuity, momentum, angular momentum, and constitutive equations, which are written as

$$
\begin{aligned}
& v_{j, j}=0, \\
& \rho D v_{i} / D t=\left(\varepsilon_{\perp} E_{j}+\Delta \varepsilon n_{k} E_{k} n_{j}\right) E_{i, j}-p_{, i}+\tau_{j i, j} \\
& \quad \begin{array}{l}
\Delta \varepsilon n_{j} E_{j} E_{i}+\gamma n_{i}-\left(\alpha_{3}-\alpha_{2}\right) N_{i}-\left(\alpha_{6}-\alpha_{5}\right) A_{i j} n_{j}-\partial F / \partial n_{i} \\
\quad+\left(\partial F / \partial n_{i, j}\right)_{, j}=0
\end{array} \\
& \begin{aligned}
\tau_{j i}= & \alpha_{1} A_{k l} n_{k} n_{l} n_{j} n_{i}+\alpha_{2} n_{j} N_{i}+\alpha_{3} N_{j} n_{i}+\alpha_{4} A_{j i}+\alpha_{5} n_{j} n_{k} A_{k i} \\
& +\alpha_{6} A_{j k} n_{k} n_{i}-\left(\partial F / \partial n_{k, j}\right) n_{k, i} .
\end{aligned}
\end{aligned}
$$

In these equations, $\mathbf{v}$ is the velocity vector, $\rho$ the fluid density, $p$ the pressure, $D / D t$ the material time derivative, $\mathbf{n}$ the director indicating the average of the directions of liquid crystal molecules, $\mathbf{E}$ the electric field vector, $\varepsilon_{\perp}$ and $\varepsilon_{\|}$the dielectric constants perpendicular and parallel to the director, respectively, $\Delta \varepsilon=\varepsilon_{\|}-\varepsilon_{\perp}$ the anisotropy of the dielectric con-

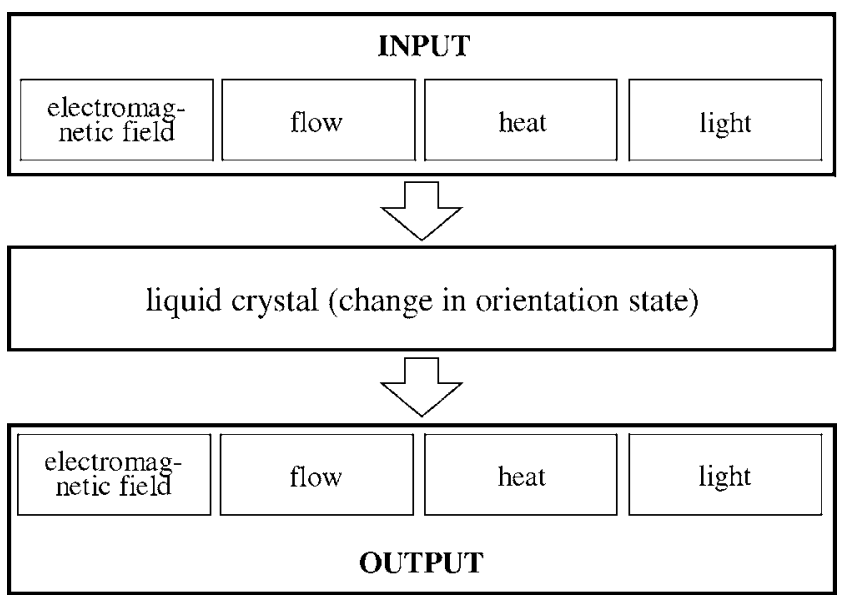

FIG. 1. Application of liquid crystal. 

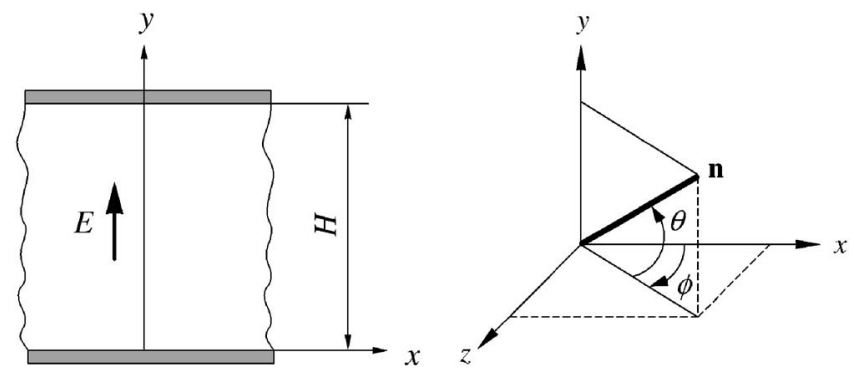

FIG. 2. Coordinate systems.

stants, A the rate of the deformation tensor, $\mathbf{N}$ the angular velocity of the director relative to that of the surrounding fluid, $\alpha_{1}-\alpha_{6}$ the Leslie viscosities, and $F$ the free energy density due to the spatial distortion of the director field expressed as

$$
2 F=K_{1}(\nabla \cdot \mathbf{n})^{2}+K_{2}(\mathbf{n} \cdot \nabla \times \mathbf{n})^{2}+K_{3}|\mathbf{n} \times \nabla \times \mathbf{n}|^{2} .
$$

Here, $K_{1}, K_{2}$, and $K_{3}$ are the elastic constants representing the splay, twist, and bend deformations of the director, respectively. $\gamma$ in Eq. (3) is required because of the constraint of director unit length.

A liquid crystal was placed between two parallel plates with the gap $H$, as shown in Fig. 2, and at time $t=0$, a uniform electric field with the intensity $E$ (=constant) was imposed in the $y$ direction. A no-slip condition for the velocity field was applied to the plates. On the other hand, for the orientation field, the tilt angle was fixed at $1^{\circ}$ and the twist angles were set at $0^{\circ}$ and $180^{\circ}$ in the present computation, where the tilt angle is $\theta$ at the plates and the twist angle is defined as the difference in $\phi$ between both plates when we express the posture of the director with $\theta$ and $\phi$, as shown in Fig. 2. For initial values, we set $\mathbf{v}=0$ in the entire domain and chose a one-dimensional steady-state solution without an electric field for director orientation. A finite difference method and a second-order Runge-Kutta method were used to discretize the governing equations. From a preliminary computation, we selected $\Delta y=H / 100$ and $\Delta t=10^{-9} \mathrm{~s}$. In this computation, we used the material constants of 4-n-pentyl$4^{\prime}$-cyanobipheny $(5 \mathrm{CB})$, which is a low-molar-mass nematic liquid crystal. The material constants are as follows; ${ }^{10-13}$ the Leslie viscosity coefficients are $\alpha_{1}=0, \alpha_{2}=-0.086$, $\alpha_{3}=-0.004, \alpha_{4}=0.089, \alpha_{5}=0.059$, and $\alpha_{6}=-0.031 \mathrm{~Pa} \mathrm{~s}$, the elastic constants are $K_{1}=6.37 \times 10^{-12}, K_{2}=3.81 \times 10^{-12}$, and $K_{3}=8.6 \times 10^{-12} \mathrm{~N}$, and the dielectric constants are $\varepsilon_{\|}=15.7$ $\times 10^{-11}$ and $\varepsilon_{\perp}=5.7 \times 10^{-11} \mathrm{~F} / \mathrm{m}$. The density $\rho$ is $1000 \mathrm{~kg} / \mathrm{m}^{3}$.

Figure 3 shows the transient velocity profiles for

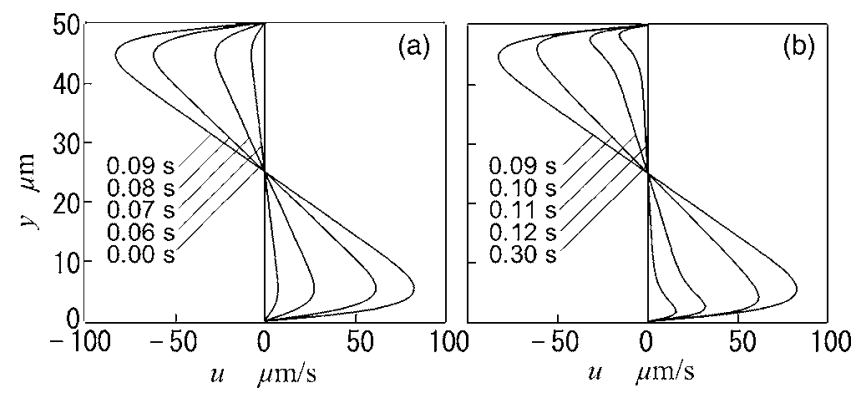

FIG. 3. Change in velocity profile with time (twist angle $=0^{\circ}$ ): (a) from start

to maximum and (b) from maximum to cessation.
Downloaded 13 Nov 2010 to 222.229.64.201. Redistribution subject to AIP license or copyright; see http://apl.aip.org/about/rights_and_permissions

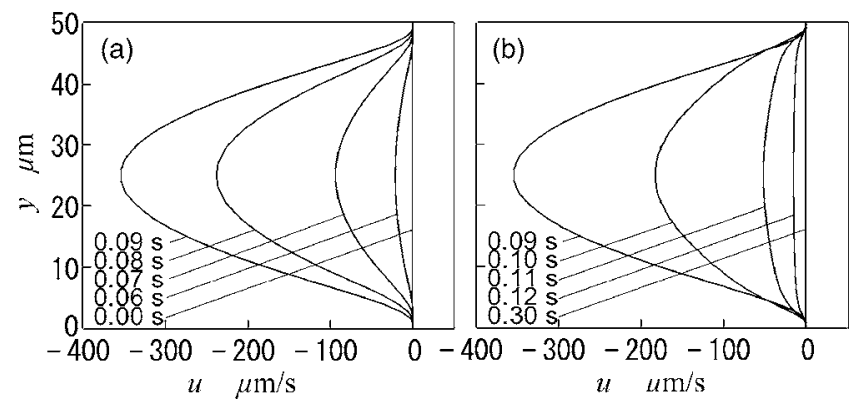

FIG. 4. Change in velocity profile with time (twist angle $=180^{\circ}$ ): (a) from start to maximum and (b) from maximum to cessation.

$H=50 \mu \mathrm{m}$ and $\phi=0^{\circ}$ when we imposed a direct-current voltage of $10 \mathrm{~V}$ at $t=0$ and kept the value constant. Figures 3(a) and 3(b) show the change from the start to the maximum and that from the maximum to the cessation, respectively. The induced velocity is negative in the upper half region and positive in the lower half region; thus, the profile is $\mathrm{S}$ shaped and the bulk flow rate is zero. A series of such changes occur in $0.3 \mathrm{~s}$ with an acceleration time of about $0.1 \mathrm{~s}$ and a deceleration time due to viscosity of about $0.2 \mathrm{~s}$. The difference in velocity profile between the acceleration and deceleration periods is small. Almost no flow occurs in the early stage of the acceleration period $t=0-0.06 \mathrm{~s}$, and the flow is rapidly accelerated for $t=0.06-0.09 \mathrm{~s}$. Similarly, a rapid deceleration occurs for $t=0.09-0.12 \mathrm{~s}$, and afterward, the flow is decelerated very slowly for $t=0.18-0.30 \mathrm{~s}$. The maximum induced velocity is approximately $80 \mu \mathrm{m} / \mathrm{s}$. Figure 4 shows the result for the twist angle of $180^{\circ}$. The flow is in the negative $x$ direction at every $y$ position; thus, the bulk flow rate is not 0 . Although the time required for the change in velocity is completely the same as that in Fig. 3, the absolute induced velocity is very large and its maximum reaches about $350 \mu \mathrm{m} / \mathrm{s}$. To confirm the flow generation, we carried out a visualization experiment using $5 \mathrm{CB}$ enclosed between two parallel glass plates with a gap of $50 \mu \mathrm{m}$. The twist angle is $0^{\circ}$ and the imposed voltage is $10 \mathrm{~V}$. Small polystyrene particles whose diameter is $2.5 \mu \mathrm{m}$ are mixed in 5CB. Figures 5(a) and 5(b) show the microscopic images obtained before and after the imposition of the voltage, respectively. The microscope focus is set to be $7.5 \mu \mathrm{m}$ below the upper plate. We can recognize the movement of a particle to the upper part by $5.6 \mu \mathrm{m}$; thus, it is confirmed experimentally that the change in orientation due to the imposition of the electric field induces a flow.

Now, let us discuss the mechanism of the flow generation. The rotation of rodlike molecules (actually the director, which is the aggregation of molecules) is considered to induce a velocity gradient. When an electric field is imposed on molecules that are oriented to the $x$ direction with a twist

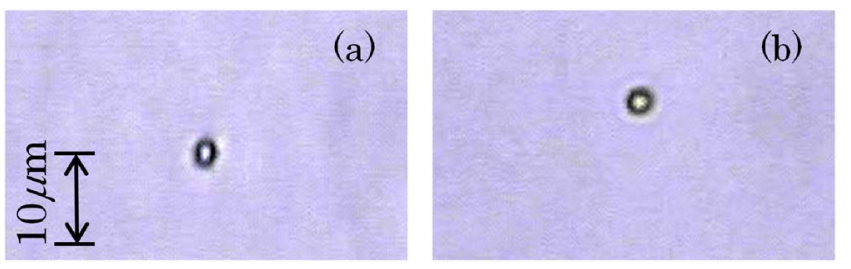

FIG. 5. (Color online) Visualization of flow induced by imposition of electric field: (a) before imposition of electric field and (b) after imposition of electric field. A small particle moved to the upper part 

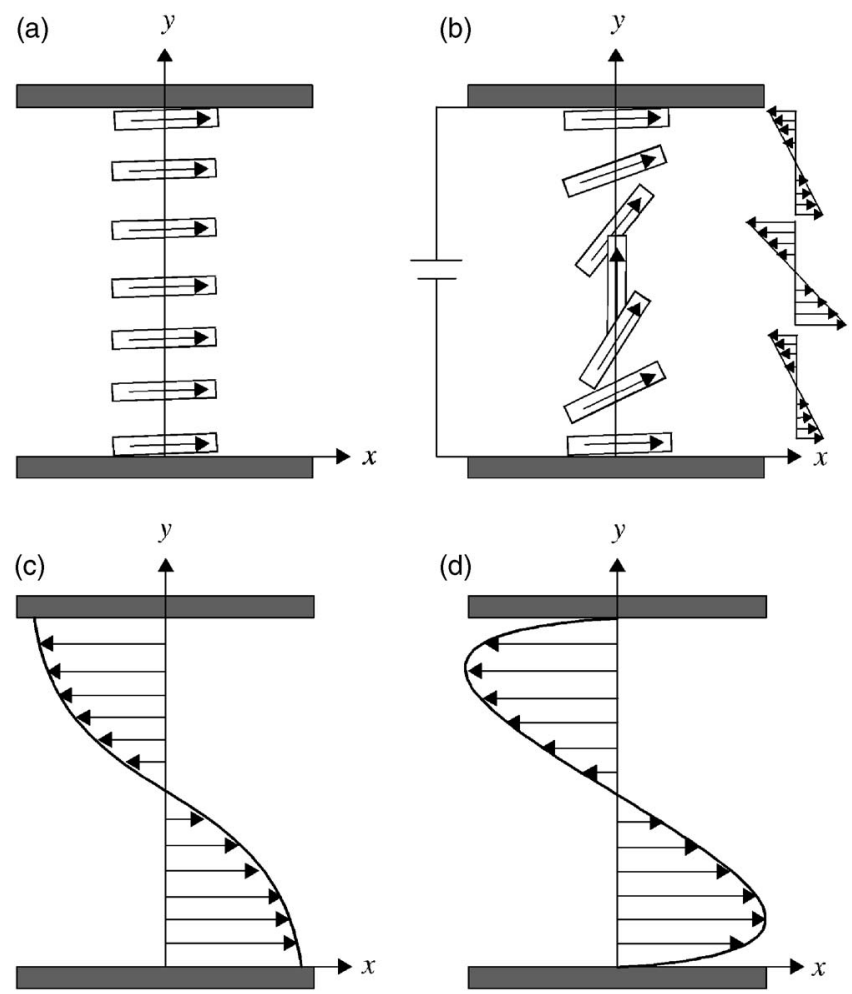

FIG. 6. Mechanism of flow generation for twisted angle of $0^{\circ}$.

angle of $0^{\circ}$, as shown in Fig. 6(a), molecules near walls rotate at a small angle and induce a small velocity gradient because of an anchoring effect, and molecules in the middle region of a channel rotate at a large angle and induce a large velocity gradient because of a small anchoring effect, as shown in Fig. 6(b). As a result, the velocity profile shown in Fig. 6(c) is obtained by integrating these velocity gradients in the $y$ direction, and finally, we obtain the profile shown in Fig. 6(d) by incorporating the no-slip condition at the walls into the profile in Fig. 6(c). We can control the rotation direction of molecules by adding a tilt angle to molecules at the walls. Figure 6 shows a case in which molecules rotate counterclockwise. On the other hand, for the twist angle of $180^{\circ}$ shown in Fig. 7(a), molecules in the lower half region of the channel rotate counterclockwise, whereas those in the upper half region rotate clockwise when an electric field is imposed on a liquid crystal. Molecules in the middle region of the channel also rotate, but such rotation does not contribute to the $x$ component of the flow, because the rotation occurs in the $y-z$ plane. As a result, the one-directional flow shown in Figs. 7(c) and 7(d) is obtained.

As mentioned above, it can be confirmed numerically and experimentally that the imposition of an electric field on a liquid crystal induces a flow. If we utilize this phenomenon, we can develop various microactuators (it is noted that the size of the actuators considered here should be more than several $10 \mathrm{~nm}$ because the present study is based on continuum mechanics). For example, a device composed of two parallel flat plates, in which one plate is fixed and the other is movable in its plane, operates as an actuator for moving a plate or a precise controller. Furthermore, by considering that a liquid crystal behaves like a liquid, liquid crystalline actuators have shape adaptability; that is, we can develop a motor if we prepare two concentric cylinders and fill the gap with a liquid crystal. ${ }^{14}$ The actuators mentioned here are only a few
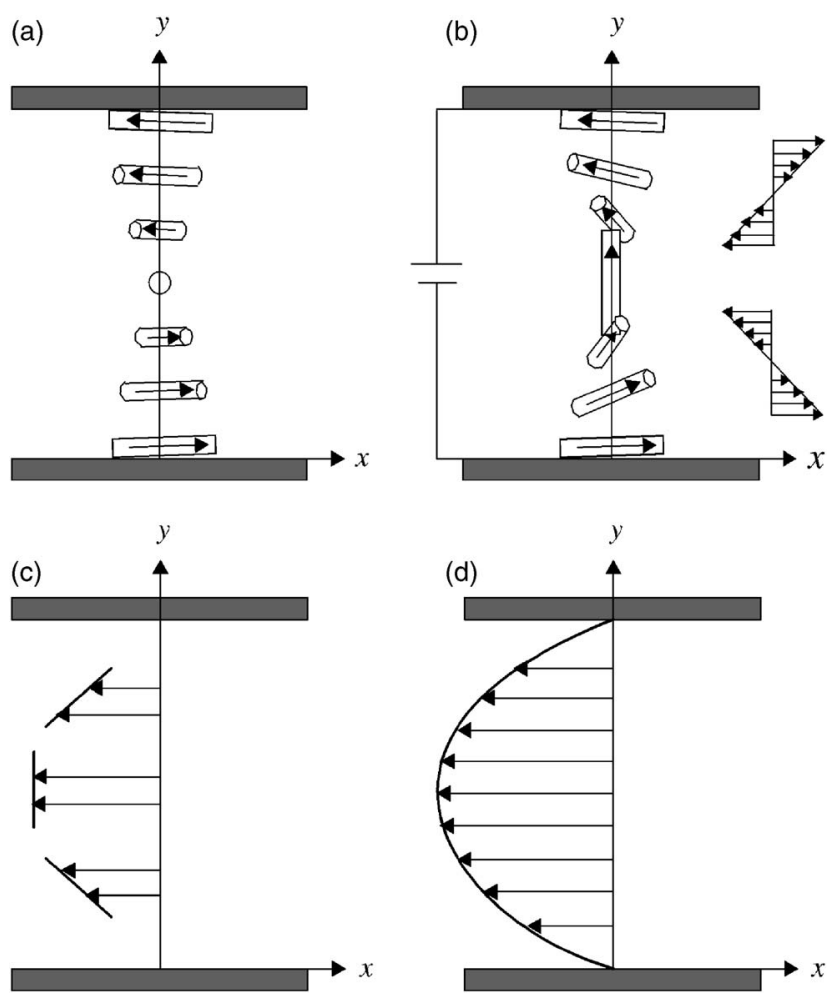

FIG. 7. Mechanism of flow generation for twisted angle of $180^{\circ}$

examples and we expect various studies on the mechanics or dynamics of liquid crystals. Furthermore, the actuator sensitivity and the effect of the material properties on the actuator performance are the problems to be solved in the near future.

In conclusion, we have computed the unsteady behavior of a liquid crystal between parallel plates and carried out a visualization experiment. As a result, it is confirmed that the imposition of an electric field on the liquid crystal induces a flow. The velocity profile depends on the twist angle. The rotation of liquid crystalline molecules induces a velocity gradient locally. That is the mechanism of the flow generation.

The authors thank financial support from a Grant-in-Aid for Scientific Research (B) and the Casio Co. Ltd. promotion foundation.

${ }^{1}$ A. D. Rey and M. M. Denn, J. Non-Newtonian Fluid Mech. 27, 375 (1988).

${ }^{2}$ S. Chono, T. Tsuji, and M. M. Denn, J. Non-Newtonian Fluid Mech. 79, 515 (1998).

${ }^{3}$ K. Koyama, Development of Electro-Rheological (ER) Fluids (CMC, Tokyo, 1999) [in Japanese].

${ }^{4}$ A. D. Rey and M. M. Denn, Annu. Rev. Fluid Mech. 34, 233 (2002).

${ }^{5}$ F. Brochard, Mol. Cryst. Liq. Cryst. 23, 51 (1973).

${ }^{6}$ D. W. Berreman, J. Appl. Phys. 46, 3746 (1975).

${ }^{7}$ J. L. Ericksen, Arch. Ration. Mech. Anal. 4, 231 (1960).

${ }^{8}$ J. L. Ericksen, Trans. Soc. Rheol. 5, 23 (1961).

${ }^{9}$ F. M. Leslie, Arch. Ration. Mech. Anal. 28, 265 (1968).

${ }^{10}$ H. Kneppe, F. Schneider, and N. K. Sharma, J. Chem. Phys. 77, 3203 (1982).

${ }^{11}$ H. Kneppe, F. Schneider, and N. K. Sharma, Ber. Bunsenges. Phys. Chem. 85, 784 (1981).

${ }^{12}$ P. P. Karat and N. V. Madhusudana, Mol. Cryst. Liq. Cryst. 40, 239 (1977).

${ }^{13}$ Editors for handbook of liquid crystals, Handbook of Liquid Crystals (Maruzen, Tokyo, 2000), p. 209.

${ }^{14}$ S. Chono and T. Tsuji, Japan Patent No. 3586734 (20 August 2004) [in Japanese]. 